\title{
The Role of Nasal Nitric Oxide and Anterior Active Rhinomanometry in the Diagnosis of Allergic Rhinitis and Asthma: A Message for Pediatric Clinical Practice
}

This article was published in the following Dove Press journal:

Journal of Asthma and Allergy

\section{Giulia Brindisi (D) ${ }^{1, *}$ \\ Valentina De Vittori ${ }^{1, *}$ \\ Rosalba De Nola (iD) 2,3 \\ Antonio Di Mauro (iD ${ }^{4}$ \\ Giovanna De Castro' \\ Maria Elisabetta Baldassarre (iD ${ }^{4}$ \\ Ettore Cicinelli ${ }^{2}$ \\ Bianca Cinicola' \\ Marzia Duse' \\ Anna Maria Zicari ${ }^{1}$ \\ 'Pediatrics Department, Umberto I Hospital, Sapienza University, Rome, 0016I, Italy; ${ }^{2}$ Department of Biomedical Science and Human Oncology, Gynecology and Obstetrics Section, "Aldo Moro" University of Bari, Bari, 70124, Italy; ${ }^{3}$ Department of Tissues and Organs Transplantation and Cellular Therapies, "Aldo Moro" University of Bari, Bari, 70124, Italy; ${ }^{4}$ Department of Biomedical Sciences and Human Oncology-Neonatology and Neonatal Intensive Care Unit, "Aldo Moro" University of Bari, Bari, 70I24, Italy}

*These authors contributed equally to this work
Correspondence: Giulia Brindisi Pediatrics Department, Umberto I Hospital, Sapienza University, Viale Regina Elena 324, Rome, 0016I, Italy Tel +3906 49979333

Email giulia.brindisi@gmail.com
Background: Allergic rhinitis (AR) and asthma are two common atopic diseases, often associated with a common ethiopathogenesis characterized by a Th2 inflammatory response with the release of many biomarkers, such as nitric oxide (NO).

Purpose: To evaluate and compare inflammatory (nFeNO and eFeNO) and functional (mNF and FEV1) parameters in AR children with or without asthma in comparison to controls. Secondly, we aimed to identify nFeNO cut-off values and verify their reliability to predict the presence of rhinitis or asthma alone or in combination.

Patients and Methods: We enrolled 160 children (6-12 years of age) with AR and/or asthma divided into four groups: controls, AR, asthma, and AR + asthma. All children underwent the following inflammatory and functional measurements: $\mathrm{nFeNO}, \mathrm{eFeNO}, \mathrm{mNF}$ and FEV1.

Results: We observed that levels of $\mathrm{nFeNO}$ were extremely higher in children with $\mathrm{AR}$ and even more in those with AR + asthma in respect to controls. Notably, all the pathological conditions, especially AR + asthma, showed significantly lower values of mNF compared to healthy children. A negative correlation linked $\mathrm{mNF}$ and $\mathrm{nFeNO}$. Then, we found eFeNO values significantly higher in all the pathological groups compared to controls, with major values of this marker in patients affected by asthma and AR + asthma, as well as FEV1 values significantly lower in all the disease groups, especially in children with asthma and $\mathrm{AR}+$ asthma. ROC curve analysis showed that $\mathrm{nFeNO}$ was a great predictor for rhinitis alone or with asthma, revealing an accurate cut-off of $662 \mathrm{ppb}$.

Conclusion: nFeNO measurement is non-invasive, easy to perform, economic and a valuable test in case of AR alone or in association with asthma. Thus, it should be used in patients with rhinitis, together with anterior active rhinomanometry (AAR) to diagnose and estimate the degree of nasal obstruction but also in children with asthma to assess their nasal involvement and improve the therapeutic management.

Keywords: nasal nitric oxide, exhaled nitric oxide, anterior active rhinomanometry, allergic rhinitis, asthma, children

\section{Introduction}

Allergic rhinitis (AR) and asthma are two common atopic diseases, commonly associated. Still too often, however, AR is an underestimated symptom at the onset, which is mostly taken into account when associated with asthma or when it becomes chronic. ${ }^{1}$ AR can lead to chronic complications, affecting negatively the quality of life of 
children and their school performances. ${ }^{2,3}$ Epidemiological studies show a continuous increase in AR prevalence worldwide reaching up to $40 \%$ in children. Thus, it represents an emerging problem in public health. ${ }^{4}$ The inflammation of the nasal mucosa starts as minimal, but it can become persistent after repeated and continuous exposure to aeroallergens. ${ }^{5}$ In this case, inflammation seems to increase proportionally to the degree of nasal obstruction, similarly to asthma after the exposure to allergic bronchial triggers. ${ }^{6,7}$ Since upper and lower airways represent a continuous entity, a high percentage of AR patients (up to 38\%) have also asthma, and the early and correct therapeutic management of both the diseases seems to bring mutual benefits. ${ }^{8,9}$ They share a common ethiopathogenesis, characterized by a T-helper 2 (Th2) inflammatory response with a release of many biomarkers, where nitric oxide (NO) is one of the most studied. ${ }^{10}$ The measurement of $\mathrm{NO}$ in the respiratory tract provides information on the degree of eosinophilic inflammation and it can complete the clinical and non-invasive management of allergic diseases. ${ }^{11-13}$ To this purpose, NO evaluation in AR could be useful in addition to an objective nasal flow assessment through anterior active rhinomanometry (AAR). ${ }^{14}$ The bronchial asthma management could similarly benefit from eFeNO measurement in addition to spirometry. From a clinical point of view, it is crucial to verify the correspondence between these inflammatory/functional parameters and the symptoms referred by patients. Several authors have already outlined the relevance of $\mathrm{nFeNO}$ as a marker of AR inflammation in children. ${ }^{15-17}$ Notably, they have shown that nFeNO level in AR (with or without asthma) was significantly increased than controls. ${ }^{18,19}$ However, to date, no studies have been conducted in children with allergic diseases analyzing inflammatory and functional parameters together. Therefore, the aim of our study was to evaluate and compare both inflammatory (nFeNO and eFeNO) and functional (mNF and FEV1) parameters in AR children, either in presence either in absence of asthma (subset A), compared to controls. The secondary outcome was to identify nFeNO cutoff values and verify its reliability to predict the presence of rhinitis or asthma alone or in combination.

\section{Patients and Methods}

\section{Study Design}

This is a cross-sectional study, conducted between September 2019 and February 2020, where 163 patients aged 6-12 years old were consecutively enrolled in the outpatient setting of the Allergology Pediatric Department of Policlinico Umberto I in Rome, after parents have signed informed consent. Among these, 3 children were not able to perform all the diagnostic tests of the study design and were considered as drop out. In the final analysis, entered 160 children.

Patients were divided into the following 4 groups (Figure 1):

1. Group 0 (G0) Control group: n 40 .

2. Group 1 (G1) Allergic rhinitis (AR): n 64.

3. Group 2 (G2) Asthma: n 22.

4. Group 3 (G3) Asthma and Allergic rhinitis (AR): n 34.

Children with AR and AR + Asthma entered subset A ( $\mathrm{n} \mathrm{98);} \mathrm{instead,} \mathrm{children} \mathrm{with} \mathrm{all} \mathrm{the} \mathrm{pathological}$ conditions (AR, asthma and AR+ asthma) entered subset $B$ (n 120).

The main demographic data of the enrolled children are summarized in Table 1.

The recruitment process had the following inclusion criteria:

(a) Age between 6 and 12 years;

(b) Diagnosis of persistent moderate allergic rhinitis (AR) according to ARIA criteria; ${ }^{3}$ and/or mild persistent asthma according to GINA criteria; $^{20}$

(c) Sensitization to dust mite, diagnosed by skin prick tests (SPTs);

Exclusion criteria were considered:

(a) AR and/or asthma exacerbations or acute respiratory infections in the previous month;

(b) Chronic diseases;

(c) Treatment with inhaled or nasal glucocorticoid or antihistamines in the previous 2 weeks;

(d) Hospitalization 30 days before the nFeNO measurement.

A control group of healthy children was enrolled simultaneously in our outpatient setting. It consisted of gendermatched children aged 6-12 years of age without AR, asthma and other allergies in their clinical history and physical examination. 


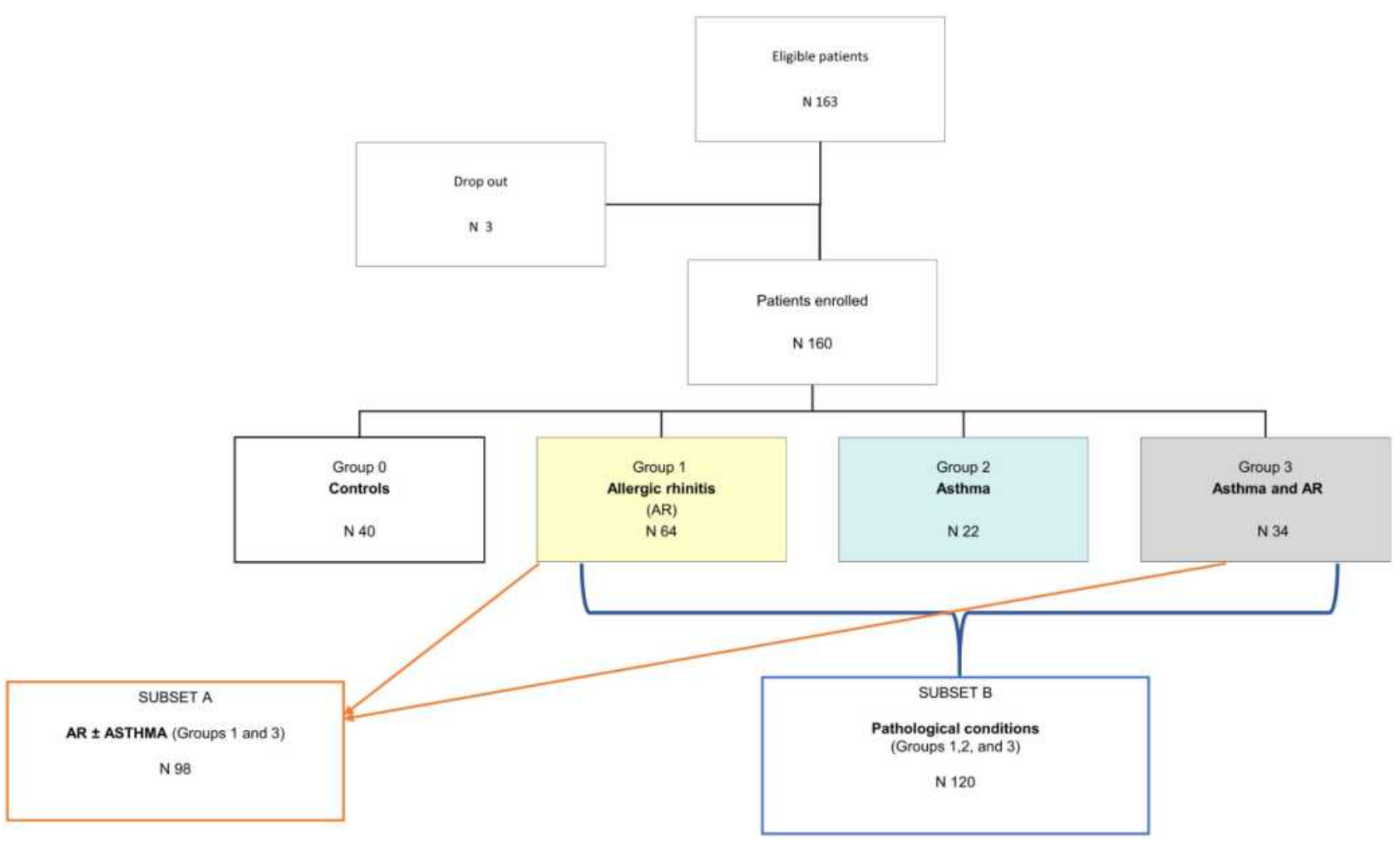

Figure I Flow chart of the study recruitment process.

The study was approved by the Bioethical Committee of Policlinico Umberto I, "Sapienza" University of Rome and conducted in accordance with the Declaration of Helsinki.
All the eligible children performed during the visit, all the following exams in the reported sequence, once for each exam:

Table I Characteristics of the Study Participants, Evaluation of Respiratory NO Metabolites and Rhinomanometric/Spirometric Values Within All Groups, Subset A and B

\begin{tabular}{|c|c|c|c|c|c|c|}
\hline & \multicolumn{4}{|l|}{ Group } & \multirow{2}{*}{$\frac{\text { Subset A }}{1+3}$} & \multirow{2}{*}{$\begin{array}{l}\text { Subset B } \\
+2+3\end{array}$} \\
\hline & 0 & I & 2 & 3 & & \\
\hline & N 40 & N 64 & N 22 & N 34 & N 98 & N 120 \\
\hline Age (years) & $8.32 \pm 1.64 *$ & $8.4 \mathrm{I} \pm \mathrm{I} .69 *$ & $8.27 \pm 1.81 *$ & $8.32 \pm 2.04 *$ & $8.38 \pm 1.81$ & $8.36 \pm 1.8^{*}$ \\
\hline Male, N (\%) & $20(50 \%)$ & $32(50 \%)$ & $13(59.1 \%)$ & 19 (55.8\%) & $5 \mathrm{I}(6 \mathrm{I} .7 \%)$ & $64(76 \%)$ \\
\hline nFeNO & $106.5[35.25] * *$ & $879.5[247.25] * *$ & $784[456.75]$ ** & $1063.5[279.75] * *$ & $966[243.50] * *$ & $899.5[240.75] * *$ \\
\hline eFeNO & $3[3] * *$ & II [3] ** & $37.5[11.25]^{* *}$ & $42.5[7] * *$ & $12.5[28] * *$ & $14.5[29.25]^{* *}$ \\
\hline FEV I & $100.17 \pm 11.61 * *$ & $96.08 \pm 8.17$ ** & $86.36 \pm 12.5 * *$ & $78.88 \pm 9.18 * *$ & $90.11 \pm 11.82 * *$ & $89.42 \pm 11.98 * *$ \\
\hline $\mathrm{mNF}$ & $94.78[\mathrm{II}]$ ** & $61.81[46.25] * *$ & $78.84[30.5]$ ** & $66.18[49] * *$ & $63.33[51.5] * *$ & $66.17[52] * *$ \\
\hline nFeNO/ eFeNO & $32.5[37.45] * *$ & $81[39.64] * *$ & $19.5[9.67]^{* *}$ & $26[9.01] * *$ & $26[57.92] * *$ & $43[57.79] * *$ \\
\hline
\end{tabular}

Notes: Summary table with demographical characteristics of the population under study and $\mathrm{nFeNO}$, eFeNO, FEVI and $\mathrm{mNF}$. Age is indicated as mean $\pm \mathrm{SD}$ and the $\mathrm{p}$-value of the one-way ANOVA F-statistic; the factorial variable is represented as numbers of males and frequency of them among each group (\%) in line with the descriptive statistics. The total of boys was 84 . The numerical variables used in our study shown with median and interquartile range [IQR], except for FEVI that is represented as mean $\pm s d$. The p-values refer to Kruskal-Wallis test for multiple comparison of all the variables, except for FEVI for which it is reported the ANOVA F-statistic's p-value since it is normal and homoscedastic. In case of single comparisons, the p-value shown results from the Wilcoxon rank-sum test for all the variables, but FEVI was studied with T student. The extremely high significant $\mathrm{p}$ values $(<0.000 \mathrm{I})$ are indicated by $* *$, whereas the non-significant ones $(\mathrm{p}$ value $>0.05)$ are identified with *. 


\section{Nasal Nitric Oxide (nFeNO)}

Measured using the exhaled nFeNO technique through a tight facemask with a fixed flow connected to an analyzer (nFeNO analyzer Medisoft), according to the American Thoracic Society/European Respiratory Society (ERS/ATS) recommendations. For each maneuver during inspiration to total lung capacity, subjects inhale through the nose from the analyzer NO-free air and then exhaled through disposable nosepieces at a constant flow of $350 \mathrm{~mL} / \mathrm{s}$ for $60 \mathrm{~s}^{13,21}$

\section{Mean Nasal Flow (mNF)}

Measured using AAR and performed according to the ICSR (Committee for the Standardization of Rhinomanometry) guidelines ${ }^{22}$ using a RINOPOCKET ED200 (EUROCLINIC ${ }^{\circledR}$, ITALY). Besides mNF, it studies nasal resistance to the air passage. The test is easy to perform and takes about 10 minutes. Rhinomanometer allows to measure the flow $\left(\mathrm{cm}^{3} / \mathrm{s}\right)$ in the right and left nostril at a pressure of 150 Pascal, in inspiration and expiration. The values obtained are compared with the pediatric values, height-dependent, reported in the literature. Nasal obstruction is classified as very severe if airflow values are lower than $29 \%$ of predicted, severe if values are between $29 \%$ and $42 \%$, moderate if values are between $43 \%$ and $56 \%$, mild for values between $57 \%$ and $70 \%$ and absent for values above $70 \% .^{14,23}$

\section{Exhaled Nitric Oxide (eFeNO)}

Measured with a Cosmed Quark NO breath device according to the procedures published for eFeNO measurement. ${ }^{13}$

\section{Forced Expiratory Volume in the Ist Second (FEVI)}

Measured with a Cosmed Spirometer, according to ATS/ ERS standards. FEV1 is the most important parameter to detect and quantify the bronchial obstruction. This value is expressed as a percentage of predicted normal values for height and gender. ${ }^{24}$

\section{Statistical Analysis}

This is a cross-sectional study. Statistical analyses were performed using the $\mathrm{R}$ statistical environment (The $\mathrm{R}$ Foundation for Statistical Computing; Vienna, Austria), specifically the packages "fBasics", "graphics", "OptimalCutpoints", "corrplot", "lawstat", and "psych". Shapiro-Wilk test and graphical evaluations of each variable were performed to demonstrate the correspondence with the normal distribution. Bartlett test was performed to evaluate the homogeneity of variances within quantitative variables resulting in FEV1, nFeNO and age as homoscedastic. Modified robust Brown-Forsythe Levene-type test, based on the absolute deviations from the median, confirmed Bartlett test's results. The categorical variables were illustrated as frequencies (\%) whereas, the statistical descriptive data were reported as the median and interquartile range [IQR] except for the two normally distributed variables (FEV1, age) that were reported separately as mean \pm standard deviation (SD). Wilcoxon ranksum test and $\mathrm{T}$ student test were used to compare each couple of groups, as appropriate. Due to the absence of homoscedasticity and normal distribution, differences between more than two groups were studied with the nonparametric Kruskal-Wallis test, also known as the oneway analysis of variance (ANOVA) on ranks, except for age and FEV1 which were analyzed with one-way ANOVA F-statistic. Correlations between numerical variables were evaluated by Pearson's coefficient using the $\mathrm{R}$ package "corrplot". In addition, the receiver-operating characteristic (ROC) curves were used to determine optimum cut-off levels of $n F e N O$ thanks to the $\mathrm{R}$ package "OptimalCutpoints". The obtained values of cut-offs and the parameters associated with them (Area under curve AUC, AUC Confidence Interval, sensitivity, specificity) were used to identify the best prediction for the presence of pathology (ie, subset B:groups 0,1,2,3) and the presence of rhinitis (ie, subset A:groups 1,3) in our population. A two-sided $\mathrm{p}$-value $<0.05$ was considered as an index of statistical significance.

\section{Results \\ Nitric Oxide Pathways and Functional Parameters in the Different Disease Groups}

Our results of NO pathways (nFeNO and eFeNO) and functional parameters ( $\mathrm{mNF}$ and FEV1) in the different disease groups are shown in the summary Tables 1 and 2 and in Figure 2.

About $n F e N O$, our data have shown a highly significant difference between all the disease groups among them and compared to controls ( $\mathrm{p}$-value $<0.0001$, Table 1). Only for the couple rhinitis versus asthma, we did not observe a significant difference (Table 2). Moreover, we observed an extremely significant difference (p-value < 
Table 2 Post Hoc Analysis of nFeNO, mNF, eFeNO, and FEVI between Groups

\begin{tabular}{|c|c|c|c|}
\hline nFeNO & Controls & AR & Asthma \\
\hline AR & $<0.0001$ & - & \\
\hline ASTHMA & $<0.0001$ & 0.22 & - \\
\hline$A R+A S T H M A$ & $<0.0001$ & $<0.0001$ & $<0.0001$ \\
\hline mNF & Controls & AR & Asthma \\
\hline AR & $<0.0001$ & - & \\
\hline ASTHMA & 0.0012 & 0.0120 & - \\
\hline$A R+A S T H M A$ & $<0.0001$ & 0.3623 & 0.0796 \\
\hline eFeNO & Controls & $\mathbf{A R}$ & Asthma \\
\hline AR & $<0.0001$ & - & - \\
\hline ASTHMA & $<0.0001$ & $<0.0001$ & - \\
\hline$A R+A S T H M A$ & $<0.0001$ & $<0.0001$ & 0.078 \\
\hline FEVI & Controls & AR & Asthma \\
\hline$A R$ & $<0.05$ & - & - \\
\hline ASTHMA & $<0.0001$ & $<0.001$ & - \\
\hline$A R+A S T H M A$ & $<0.0001$ & $<0.0001$ & 0.0137 \\
\hline RATIO nFeNO/eFeNO & Controls & AR & Asthma \\
\hline AR & $<0.0001$ & - & - \\
\hline ASTHMA & 0.005 & $<0.0001$ & - \\
\hline$A R+A S T H M A$ & $<0.0001$ & $<0.0001$ & 0.02 \\
\hline
\end{tabular}

Notes: Post hoc analysis for Holm's or Bonferroni (ratio nFeNO/eFeNO) p-value adjustment after Kruskal-Wallis rank-sum test of nFeNO, mNF, eFeNO, and FEVI between groups. Non-significant values $(p>0.05)$ are in italic.

0.0001) between children with $\mathrm{AR}$ and $\mathrm{AR}+$ asthma, grouped in subset $\mathrm{A}$ and controls, with higher values of $\mathrm{nFeNO}$ in all the children with AR (common condition between group 1 and 3). Since it emerged that even the patients with only asthma (group 2) had a higher value of nFeNO than controls, we decided to explore the role of $\mathrm{nFeNO}$ in all the pathologic conditions as a unique subset (subset B) compared to controls. Also, the subset B, composed of patients with all the pathological conditions together, had significantly higher $\mathrm{nFeNO}$ levels compared to healthy children (p-value $<0.0001$ ) (Table 1).

Then, we evaluated mNF between groups, finding a significant difference between all the disease groups considered alone vs controls ( $p$-value $<0.0001)$ and between asthma vs AR ( $\mathrm{p}$-value $=0.012$ ). Instead, there was no statistically significant difference between the couples $\mathrm{AR}+$ asthma vs $\mathrm{AR}$ and $\mathrm{AR}+$ asthma vs asthma (Table 2). As expected, we found the lower values of nasal flow in children with rhinitis with or without asthma (Subset A, Table 1). Anyway, patients with all the pathological conditions (subset B) showed significantly lower nasal flow values compared to healthy subjects (Table 1).

Concerning eFeNO, there were significant differences between all the groups ( $\mathrm{p}$-value $<0.0001$ ) except for children with asthma vs AR + asthma (Table 2), both groups where eFeNO presents higher values in respect to the groups without asthma. Children grouped in subset $\mathrm{A}$ and $\mathrm{B}$ had eFeNO values significantly higher in respect to controls, as showed in Table 1.

Similarly, we evidenced a statistically significant difference of FEV1 among all the groups (p-value $<0.0001$, Tables 1 and 2): asthma and AR + asthma are the groups with the lowest values of FEV1, as expected. We observed a statistically significant difference between either subset A vs controls and subset B vs controls (Table 1).

Since eFeNO seems to be more related to asthma and nFeNO to rhinitis, we also evaluated their ratio (nFeNO/ eFeNO): it emerged that the highest value characterized group 1 (AR), whereas the lowest one was found in the asthma group (group 2).

\section{ROC Curve Analysis to Evaluate Cut-off Values of $n F e N O$ for $A R$ with and without Asthma}

We performed ROC curve analyses of nFeNO in the perspective to find the best predictive index of AR with and without the contemporary presence of asthma. The ROC curve of $\mathrm{nFeNO}$ as a predictor for isolated asthma presented a low area under the curve (AUC) indicating that it cannot distinguish patients with asthma alone, whereas it behaved like a great predictor for subset A (rhinitis alone or with asthma), revealing an accurate cut-off of $662 \mathrm{ppb}$ (Figure 3A). In detail, we found two sex-adjusted cut-offs based on the Youden index: $602 \mathrm{ppb}$ for males and $662 \mathrm{ppb}$ for females (Figure $3 \mathrm{~B}$ and $\mathrm{C}$, respectively) with a slightly more accurate test for females $(\mathrm{AUC}=0.918)$ than for males $(\mathrm{AUC}=0.847)$. Notably, $\mathrm{nFeNO}$ can predict with high accuracy (Figure 4A) the presence of allergic rhinitis with asthma (without considering the only rhinitis) revealing a cut-off of $900 \mathrm{ppb}$ that increased for (990) females (Figure 4C), whereas it slightly decreased for males (870), see Figure 4B for details. Both the ROC curves (Figures 3 and 4) identified $n F e N O$ as an optimal predictor for $A R \pm$ asthma (subset A) and AR+asthma (group 3) with an AUC, respectively, of $88 \%$ and $87 \%$. However, the former had a lower cut-off probably due to the wider range considering also group 1 that had a statistically significant lower value of nFeNO than group 3 (see Tables 1 and 2). Moreover, as reported in the following paragraph, nFeNO increases as eFeNO rises with a strong positive correlation. Since eFeNO rockets in groups with asthma than in groups without 

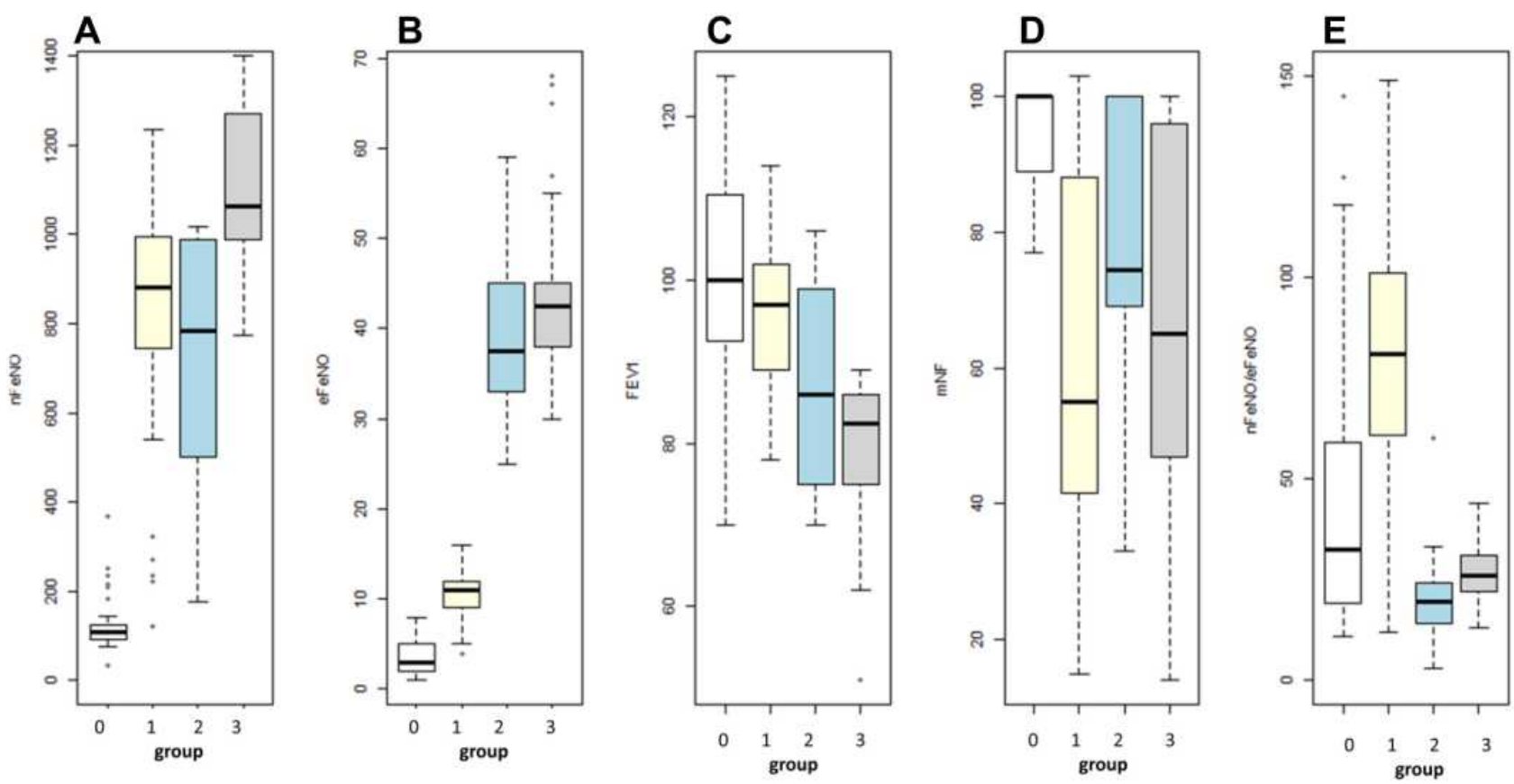

Figure 2 Boxplots of the distribution of the variables between groups. Boxplots representing the distribution of the variables $\mathrm{nFeNO}(\mathbf{A})$, eFeNO (B), FEVI (C), $\mathrm{mNF}$ (D) and $\mathrm{nFeNO} / \mathrm{eFeNO}$ (E) among the four groups of the population: white (group I, ie controls), light yellow (group 2, ie allergic rhinitis), light blue (group 3, ie asthma) and grey (group 3, ie allergic rhinitis and asthma). All the $\mathrm{p}$ values were $<0.000 \mathrm{I}$ for each variables between groups using Kruskal-Wallis rank-sum test (A-E). See also the post hoc analysis tables for each couple of the comparisons.

A ROC Curve. Criterion: Youden



\begin{tabular}{|l|l|}
\hline AUC & 0.882 \\
\hline CIAUC & $0.822,0.942$ \\
\hline CUT-OFF & 662 \\
\hline Se & 0.908 \\
\hline Sp & 0.790 \\
\hline
\end{tabular}

B Roc Curve. Criterion: Youden M

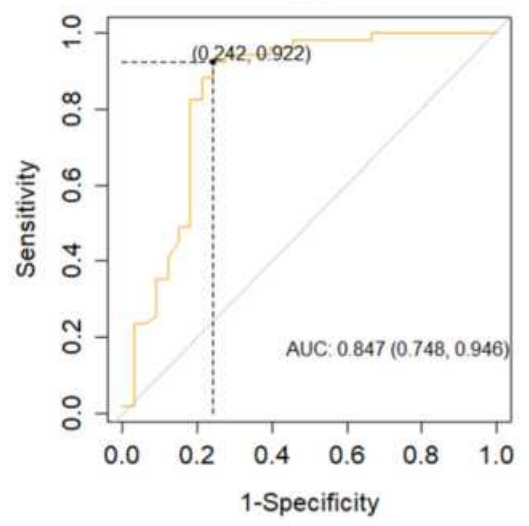

\begin{tabular}{|l|l|}
\hline AUC & 0.847 \\
\hline CI AUC & $0.748,0.946$ \\
\hline CUT-OFF & 602 \\
\hline Se & 0.922 \\
\hline Sp & 0.757 \\
\hline
\end{tabular}


\begin{tabular}{|l|l|}
\hline AUC & 0.918 \\
\hline CI AUC & $0.852,0.984$ \\
\hline CUT-OFF & 662 \\
\hline Se & 0.936 \\
\hline Sp & 0.793 \\
\hline
\end{tabular}

Figure 3 ROC curves of nFeNO for subset $A$. ROC curves of nFeNO as a predictor for allergic rhinitis alone or with asthma (subset $A)$ without the covariate sex (A). Next, two analogue sex-adjusted curves, respectively, for males (B) and females (C). All the graphs report the cut-off values of nFeNO, specificity (Sp) and sensitivity (Se) levels with also the area under curve (AUC) values and its $95 \%$ confidence interval $(\mathrm{Cl})$. Below each figure, there is the relative summary: $\mathrm{AUC}$, AUC Cl $95 \%$, Se and $\mathrm{Sp}$. 
A

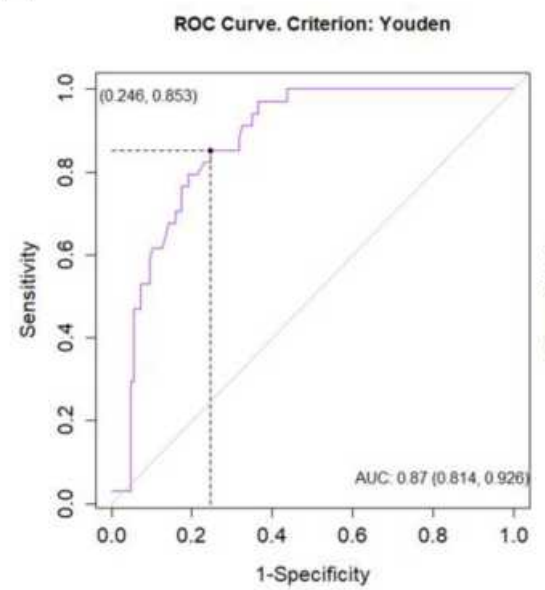

\begin{tabular}{|l|l|}
\hline AUC & 0.87 \\
\hline CI AUC & $0.814,0.926$ \\
\hline CUT-OFF & 900 \\
\hline Se & 0.853 \\
\hline Sp & 0.754 \\
\hline
\end{tabular}

B

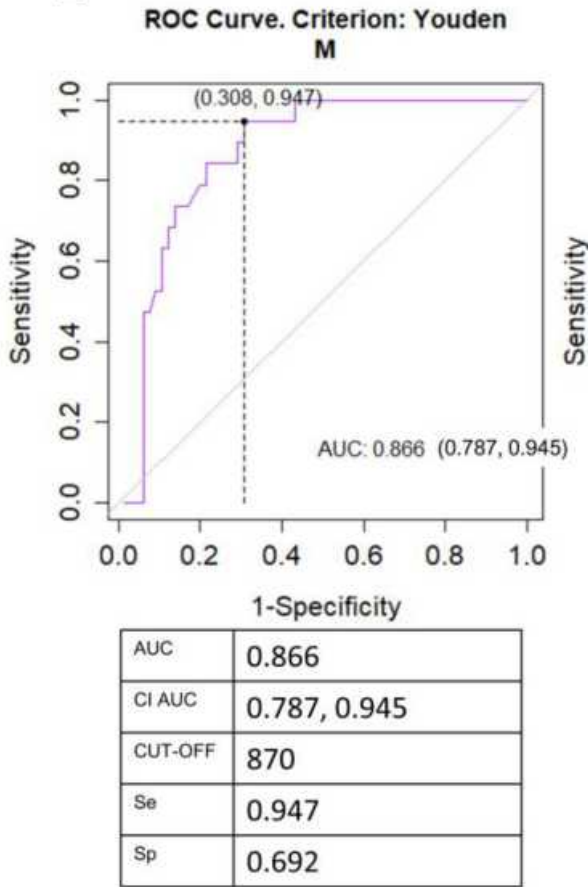

C

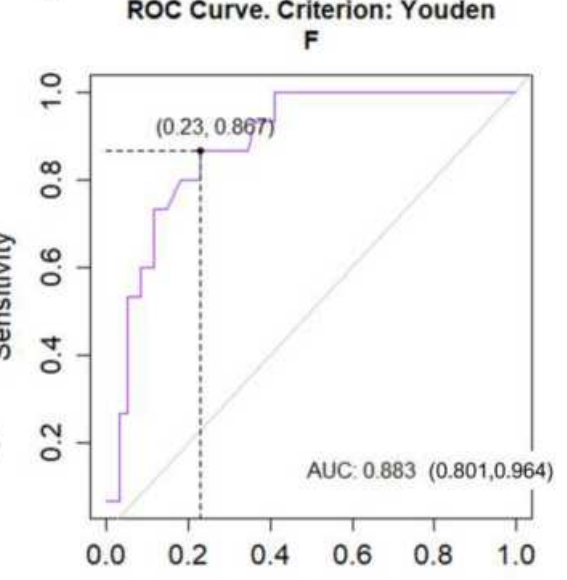

\begin{tabular}{|l|l|}
\multicolumn{2}{c}{1 -Specificity } \\
\hline AUC & 0.883 \\
\hline CI AUC & $0.801,0.964$ \\
\hline CUT-OFF & 990 \\
\hline Se & 0.866 \\
\hline Sp & 0.770 \\
\hline
\end{tabular}

Figure 4 ROC curves of $n F e N O$ for group 3. ROC curves of nFeNO as a predictor for asthma and allergic rhinitis (group 3) without the covariate sex (A). Next, two analogue sex-adjusted curves, respectively, for males (B) and females (C). All the graphs report the cut off values of nFeNO, specificity (Sp) and sensitivity (Se) levels with also the area under curve (AUC) values and its $95 \%$ confidence interval $(\mathrm{Cl})$. Below each figure, there is the relative summary: $\mathrm{AUC}$, $\mathrm{AUC} \mathrm{Cl} 95 \%$, Se and Sp.

it (see Figure 2 and Table 1), it can be reasonable that also $\mathrm{nFeNO}$ increased in groups 3 (AR +asthma) than subset A due to the presence of group 1 .

\section{Pearson Correlation Between the Main Respiratory Indexes and NO's Metabolites}

At the end, we found a strong positive Pearson correlation $(\mathrm{r}=0.50)$ between $\mathrm{nFeNO}$ and $\mathrm{eFeNO}$, a strong negative correlation $(\mathrm{r}=-0.61)$ between eFeNO and FEV1 and also $(\mathrm{r}=-0.61)$ between $\mathrm{nFeNO}$ and FEV1 (Figure 5A-C, respectively). Moreover, there was a mild negative correlation between $\mathrm{nFeNO}$ and $\mathrm{mNF}$ (Figure 5D). All the indexes obtained were statistically significant $(\mathrm{p}<0.0001)$, as shown in Figure 5. Lastly, the correlations between FEV 1 and the ratio $\mathrm{nFeNO} / \mathrm{eFeNO}$ or $\mathrm{mNF}$ and ratio $\mathrm{nFeNO} / \mathrm{eFeNO}$ were actually weak (Figure $5 \mathrm{E}$ and $\mathrm{F}$, respectively).

\section{Discussion}

The first objective of our observational study was to evaluate the functional and inflammatory parameters (nFeNO, $\mathrm{mNF}$, eFeNO and FEV1) of children with AR alone or associated with asthma, comparing these values with those of controls. Therefore, we created a subset (subset A) with children affected by AR alone and with asthma in order to explore the values of these markers in subset A evaluating allergic inflammation of upper and lower airways comparing these values with controls. Furthermore, we wanted to determine $\mathrm{nFeNO}$ cut-off values and verify its reliability to predict the presence of rhinitis or asthma alone or in combination.

Among these parameters, as known from the literature, eFeNO and FEV1 are two validated and complementary parameters for the diagnostic and therapeutic management of asthma. In detail, eFeNO is an inflammatory marker of lower airways, and although its use is debated and still controversial, ${ }^{25,26}$ according to BTS guidelines it should be mostly used to monitor the severity of asthma and the response to the treatment. ${ }^{27}$ Our data, according to previous studies, show that its levels were higher in children with asthma and even more in those with asthma and rhinitis, with a significant difference compared to healthy children, showing that the presence of asthma is the major determinant of the increase of eFeNO, that represents a biomarker of allergic asthma. We found FEV1 values significantly lower in all the disease groups in comparison 


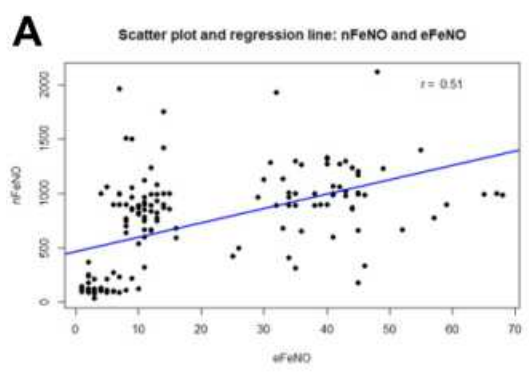

$p$ value $<0.0001$

D



p value $<0.0001$

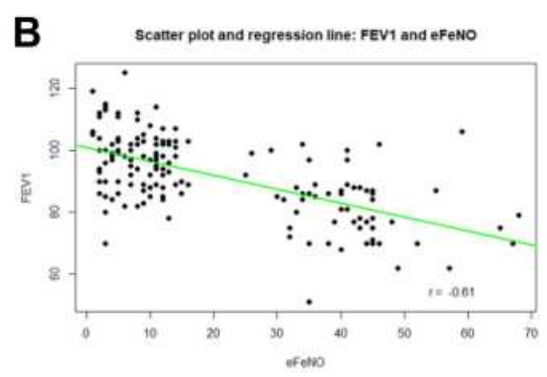

$p$ value $<0.0001$

E

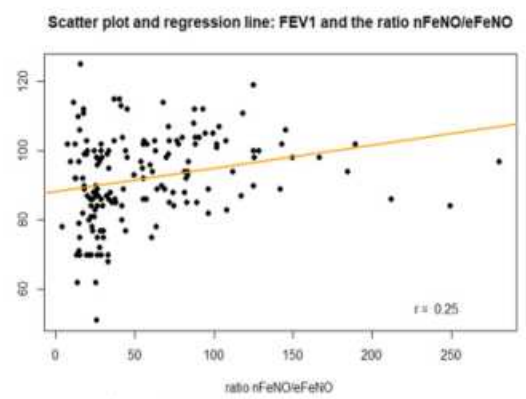

$p$ value $=0.0001$

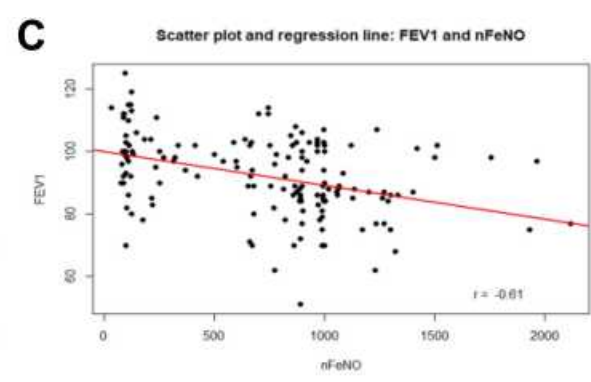

$p$ value $<0.0001$

F



Figure 5 Scatter plots and Pearson's correlation among nFeNO, mNF, eFeNO, FEVI and the ratio nFeNO/eFeNO. The scatter plots with the regression line and Pearson's correlation coefficient of the linear correlation: positive correlation $(r=-0.5 \mathrm{I})$ between $\mathrm{nFeNO}$ with eFeNO (A), negative correlation $(r=-0.6 \mathrm{I})$ between $\mathrm{FEVI}$ with eFeNO (B), negative correlation $(r=-0.61)$ between FEVI and $n F e N O(C)$, negative correlation $(r=-0.39)$ between $n F e N O$ and $m N F(D)$, positive correlation $(r=0.25)$ between $\mathrm{FEVI}$ and the ratio $\mathrm{nFeNO} / \mathrm{eFeNO}(\mathbf{E})$, and the negative correlation $(\mathrm{r}=-0.12)$ between $\mathrm{mNF}$ and the ratio $\mathrm{nFeNO} / \mathrm{eFeNO}(\mathbf{F})$.

Abbreviation: NS, non-significant.

to healthy children, especially in patients with AR + asthma (group 3). The combination of both conditions (rhinitis and asthma) worsens FEV1 and eFeNO values. $^{2,5}$ Furthermore, FEV1 and eFeNO had in our patients a negative correlation (Figure 5B), showing that a more severe bronchial obstruction corresponds to a greater degree of airway inflammation.

Concerning nasal functionality, the use of AAR has been recently validated for $\mathrm{AR}$ in children ${ }^{14}$ and it represents an objective method for the assessment of nasal obstruction in these patients. $\mathrm{nFeNO}$ is, instead, a marker of nasal allergic inflammation, and its measurement is easy to perform, non-invasive, painless and suitable for an immediate clinical evaluation in the outpatient setting. These exams are possible in compliant children from 6 years of age. ${ }^{13}$ However, the debate on the utility of nFeNO in allergic diseases is still open, especially for the presence of different and not standardized methods of measurement, which causes difficulties in comparing studies among each other. ${ }^{16}$

To date, according to the ATS/ERS recommendations, the only clinical application of $\mathrm{nFeNO}$ is limited to primary ciliary dyskinesia (PCD). ${ }^{13}$ The possible role of this biomarker has been also studied in chronic rhinosinusitis; a recent review of several studies, conducted mainly in adults, pointed out that inflammation of sino-nasal mucosa, especially if associated with polyps, prevents NO to flow from sinuses to the nasal lumen so that reduced $\mathrm{nFeNO}$ levels are measured in these conditions. ${ }^{28}$

Despite the need to further define the measurement of this biomarker, it could be of great help in AR to distinguish the inflammatory or mechanical etiology of rhinitis and to assess the response to nasal treatments. ${ }^{29}$

So far, there are no pediatric studies that have evaluated both inflammatory and functional parameters in children with $\mathrm{AR}$ and/or asthma. Takeno et $\mathrm{al}^{30}$ studied adults with untreated $\mathrm{AR}$, reporting higher levels of $\mathrm{nFeNO}$ in comparison to controls without significant difference in the resistance to nasal airflow, concluding that nFeNO and AAR are two independent measures for the objective evaluation of nasal functions.

Chen et $\mathrm{al}^{31}$ studied children with asthma, finding higher eFeNO levels and lower nasal flow in subjects with more severe nasal obstruction. They conclude that rhinomanometry is a useful tool for children with asthma to complete their diagnostic process in addition to eFeNO. Notably, the authors in this study did not consider $\mathrm{nFeNO}$. 
From the analysis of nasal parameters in our children, we detected lower $\mathrm{mNF}$ values in all the disease groups, mostly in children with AR alone followed by those with $\mathrm{AR}$ and asthma compared to controls. Hence, the presence of rhinitis causes alone a significant reduction of $\mathrm{mNF}$. Moreover, we detected significantly higher $\mathrm{nFeNO}$ levels mostly in children with AR + asthma and in those with AR alone in respect to controls, in accordance with the already published studies. ${ }^{18,19}$ This finding reinforces the role of asthma in worsening also nasal inflammation thanks to the connection between upper and lower airways. Furthermore, we found a negative correlation between $\mathrm{mNF}$ and $\mathrm{nFeNO}$, indicating that the lower the nasal flow, the higher the nasal inflammation (Figure 5D). So based on our experience, $\mathrm{nFeNO}$ and $\mathrm{mNF}$ are two complementary tools, useful to perform a more detailed diagnosis of AR.

The correlations between FEV1 or mNF and the ratio $\mathrm{nFeNO} / \mathrm{eFeNO}$ were too weak to be clinically considered, probably due to the number of children enrolled. Moreover, the former one (FEV1 and $\mathrm{nFeNO} / \mathrm{eFeNO}$ ) was statistically not significant. From our data emerged an analogue negative correlation between eFeNO and mNF already demonstrated by Chen et al. ${ }^{31}$ Even if the associated p-value is significative (p-value: 0.02 ), the correlation index $(\mathrm{r}=-0.18)$ is too weak for clinical purposes (not shown). As we are planning for further studies, the power of these correlations might benefit from a larger sample size.

Furthermore, we found a positive correlation between $\mathrm{eFeNO}$ and $\mathrm{nFeNO}$ (Figure 5A), reinforcing once again the concept of "one way one disease" in allergic patients, with an anatomical and inflammatory continuity of upper and lower airways. The combination of these methods, eFeNO, nFeNO and FEV1, could help to early detect and manage allergic children with both AR and asthma, evaluating nasal and bronchial inflammation.

Finally, from cut-off values obtained by the ROC-curve analysis, we observed a greater reliability (with high sensitivity and specificity) of $\mathrm{nFeNO}$ in distinguishing $\mathrm{AR}$ with or without asthma. Moreover, we identified genderadjusted cut-off values giving the possibility to perform a more reliable test both for boys and girls. However, the differences between the two genders need further studies, with a wider sample size, to be thoroughly explained. Similarly, Galianik et $\mathrm{al}^{18}$ studied $\mathrm{nFeNO}$ cut-off values in allergic children finding that this marker presents high sensitivity and specificity to detect AR with and without asthma in respect to controls.

The present study has some limitations. Firstly, the sample size for each group is quite small and it was all enrolled from the same outpatient setting, so other studies are necessary to confirm our observations. Moreover, $\mathrm{nFeNO}$ technique of measurement is not yet standardized and it needs improvements in methodology to become a completely reliable tool, comparable between different studies.

\section{Conclusion}

In AR children, especially those with asthma, nFeNO levels are higher than controls and high levels of nasal inflammation are related to a lower mNF. This shows that $\mathrm{nFeNO}$ and AAR are complementary tools in the diagnosis of AR. Similarly, high levels of eFeNO indicative of bronchial inflammation correlate with a lower FEV1. The well-known concept of the anatomical and functional continuity between upper and lower airways is further confirmed by the linear correlation between $\mathrm{nFeNO}$ and eFeNO. The measurement of $\mathrm{nFeNO}$ is non-invasive, easy to perform and economic and it should be used in patients with rhinitis, together with AAR, to diagnose and estimate the degree of nasal obstruction but also in children with asthma to assess their nasal involvement and improve the therapeutic management.

\section{Disclosure}

The authors report no conflicts of interest in this work.

\section{References}

1. Klimek L, Bachert C, Pfaar O. ARIA guideline 2019: treatment of allergic rhinitis in the German health system. Allergol Sel. 2019;3 (1):22-50. doi:10.5414/ALX02120E

2. Cipriani F, Tripodi S, Panetta V, et al. Early molecular biomarkers predicting the evolution of allergic rhinitis and its comorbidities: a longitudinal multicenter study of a patient cohort. Pediatr Allergy Immunol. 2019;30(3):325-334. doi:10.1111/pai.13036

3. Brożek Jan L, Bousquet J, Agache I, et al. Allergic Rhinitis and Its Impact on Asthma (ARIA) guidelines-2016 revision. J Allergy Clin Immunol. 2017;140(4):950-958. doi:10.1016/j.jaci.2017.03.050

4. Mallol J, Crane J, von Mutius E, et al. The International Study of Asthma and Allergies in Childhood (ISAAC) phase three: a global synthesis. Allergol Immunopathol. 2013;41(2):73-85. doi:10.1016/j. aller.2012.03.001

5. Schuler ICF, Montejo JM. Allergic rhinitis in children and adolescents. Pediatr Clin North Am. 2019;66(5):981-993. doi:10.1016/j. pcl.2019.06.004

6. Agache I, Lau S, Akdis CA, et al. EAACI guidelines on allergen immunotherapy: house dust mite-driven allergic asthma. Allergy. 2019;74(5):855-873. doi:10.1111/all.13749 
7. Bush A. Pathophysiological mechanisms of asthma. Front Pediatr. 2019;19:68. doi:10.3389/fped.2019.00068

8. van Vliet D, Essers BA, Winkens B, et al. Longitudinal relationships between asthma-specific quality of life and asthma control in children; the influence of chronic rhinitis. J Clin Med. 2020;9(2):555. doi: $10.3390 /$ jcm 9020555

9. De Vittori V, Pacilio A, Indinnimeo L, et al. When asthma and rhinitis coexist, could rhinitis reduce asthma control in children? Allergy Asthma Proc. 2019;40(3):210. doi:10.2500/aap.2019.40.4219

10. Dweik RA, Boggs PB, Erzurum SC, et al. An official ATS clinical practice guideline: interpretation of exhaled nitric oxide levels (FENO) for clinical applications. Am J Respir Crit Care Med. 2011;184:602-615. doi:10.1164/rccm.9120-11ST

11. Hoyte FCL, Gross LM, Katial RK. Exhaled nitric oxide: an update. Immunol Allergy Clin North Am. 2018;38(4):573-585. doi:10.1016/j. iac.2018.06.001

12. Ambrosino P, Parrella P, Formisano R, et al. Clinical application of nasal nitric oxide measurement in allergic rhinitis: a systematic review and meta-analysis. Ann Allergy Asthma Immunol. 2020: S1081-1206(20)30479-8. doi:10.1016/j.anai.2020.07.003

13. American Thoracic Society, European Respiratory Society. ATS/ERS recommendations for standardized procedures for the online and offline measurement of exhaled lower respiratory nitric oxide and nasal nitric oxide, 2005. Am J Respir Crit Care Med. 2005;171 (8):912-930. doi:10.1164/rccm.200406-710ST

14. Cilluffo G, Zicari AM, Ferrante G, et al. Assessing repeatability and reproducibility of Anterior Active Rhinomanometry (AAR) in children. BMC Med Res Methodol. 2020;20(1):86. doi:10.1186/ s12874-020-00969-1

15. Chen QB, Chen L, Zheng XY, et al. The clinical significance of nasal nitric oxide concentrations in preschool children with nasal inflammatory disease. Int Arch Allergy Immunol. 2020;181(1):51-55. doi:10.1159/000504192

16. Wang PP, Wang GX, Ge WT, et al. Nasal nitric oxide in allergic rhinitis in children and its relationship to severity and treatment. Allergy Asthma Clin Immunol. 2017;13:20.

17. Liu D, Huang Z, Huang Y, et al. Measurement of nasal and fractional exhaled nitric oxide in children with upper airway inflammatory disease: preliminary results. Int $J$ Pediatr Otorhinolaryngol. 2015;79(12):2308-2311. doi:10.1016/j.ijporl.2015.10.033

18. Galiniak S, Biesiadecki M, Aebisher D, et al. Nasal nitric oxide in upper airways in children with asthma and allergic rhinitis. Adv Med Sci. 2020;65(1):127-133. doi:10.1016/j.advms.2019.11.005
19. Duong-Quy S, Vu-Minh T, Hua-Huy T, et al. Study of nasal exhaled nitric oxide levels in diagnosis of allergic rhinitis in subjects with and without asthma. J Asthma Allergy. 2017;10:75-82. doi:10.2147/JAA. S129047

20. Global Initiative for Asthma (GINA). 2017. Available from: http:// ginasthma.org/archived-reports. Accessed February 27, 2021.

21. Manna A, Montella S, Maniscalco M, et al. Clinical application of nasal nitric oxide measurement in pediatric airway diseases. Pediatr Pulmonol. 2015;50(1):85-99. doi:10.1002/ppul.23094

22. Clement PAR, Gordts F. Consensus report on acoustic rhinometry and rhinomanometry. Rhinology. 2005;43:169Y179.

23. Zapletal A, Chalupová J. Nasal airflow and resistance measured by active anterior rhinomanometry in healthy children and adolescents. Pediatr Pulmonol. 2002;33(3):174-180. doi:10.1002/ppul.10066

24. Miller MR, Hankinson J, Brusasco V, et al. Standardisation of spirometry. Eur Respir J. 2005;26(2):319-338. doi:10.1183/ 09031936.05.00034805

25. Ciprandi G, Tosca MA, Cirillo I, et al. Impact of allergic rhinitis on asthma in children: effects on bronchial hyperreactivity. Allergy. 2010;65(9):1199-1201. doi:10.1111/j.1398-9995.2009.02321.x

26. Khalili B, Boggs PB, Shi R, et al. Discrepancy between clinical asthma control assessment tools and fractional exhaled nitric oxide. Ann Allergy Asthma Immunol. 2008;101(2):124-129. doi:10.1016/ S1081-1206(10)60199-8

27. British Thoracic Society. Scottish intercollegiate guidelines network. British guideline on the management of asthma. Thorax. 2014;69 (Suppl 1):1-192.

28. Ferraro VA, Zanconato S, Baraldi E, Carraro S. Nitric oxide and biological mediators in pediatric chronic rhinosinusitis and asthma. J Clin Med. 2019;8(11):1783. doi:10.3390/jcm8111783

29. Maniscalco M, Sofia M, Pelaia G. Nitric oxide in upper airways inflammatory diseases. Inflamm Res. 2007;56:58-69. doi:10.1007/ s00011-006-6111-1

30. Takeno S, Osada R, Furukido K, et al. Increased nitric oxide production in nasal epithelial cells from allergic patients - RT-PCR analysis and direct imaging by a fluorescence indicator: DAF-2 DA. Clinical \& Experimental Allergy. 2001;31(6):881-888. doi:10.1046/j.13652222.2001.01093.x

31. Chen IC, Lin YT, Hsu JH, et al. Nasal airflow measured by rhinomanometry correlates with FeNO in children with asthma. PLoS One. 2016;11(10):e0165440.

\section{Publish your work in this journal}

The Journal of Asthma and Allergy is an international, peer-reviewed open-access journal publishing original research, reports, editorials and commentaries on the following topics: Asthma; Pulmonary physiology; Asthma related clinical health; Clinical immunology and the immunological basis of disease; Pharmacological interventions and new therapies. The manuscript management system is completely online and includes a very quick and fair peer-review system, which is all easy to use. Visit http://www.dovepress.com/testimonials.php to read real quotes from published authors. 